\title{
QUANTIFICAÇÃO E VALORAÇÃO DE PRODUTOS FLORESTAIS NÃO-MADEIREIROS
}

\author{
Fabíola Gisela Pinto de Queiroz Guerra ${ }^{1}$, Anadalvo Juazeiro dos Santos ${ }^{2}$, Carlos Roberto Sanquetta ${ }^{3}$, \\ Alexandre Muzy Bittencourt ${ }^{4}$, Alexandre Nascimento de Almeida ${ }^{5}$ \\ ${ }^{1}$ Eng ${ }^{\mathrm{a}}$ Florestal, M.Sc., UFPR, Curitiba, PR, Brasil - fgpqueiroz@ig.com.br \\ ${ }^{2}$ Eng. Florestal, Dr., Depto. de Economia Rural e Extensão, UFPR, Curitiba, PR, Brasil - ajsantos@ufpr.br \\ ${ }^{3}$ Eng. Florestal, Dr., Depto. de Ciências Florestais, UFPR, Curitiba, PR, Brasil - sanquetta@ufpr.br \\ ${ }^{4}$ Eng. Florestal M.Sc., Doutorando em Engenharia Florestal, UFPR, Curitiba, PR, Brasil - alexbitten@ terra.com.br \\ ${ }^{5}$ Eng. Florestal M.Sc., UFPR, Curitiba, PR, Brasil - alexfloresta @ pop.com.br \\ Recebido para publicação: 13/09/2007 - Aceito para publicação: 20/08/2008
}

\begin{abstract}
Resumo
Atualmente, a redução da biodiversidade, em virtude do aumento do desflorestamento, fez com que se buscassem meios de se preservar os remanescentes florestais no Brasil e no mundo. Nesse contexto, os Produtos Florestais Não-Madeireiros (PFNM) tornaram-se uma alternativa para auxiliar a conservação das florestas, uma vez que, na maioria dos casos, a extração de PFNM não implica a supressão da cobertura florestal. Sendo assim, tem crescido o número de estudos sobre o potencial dos PFNM para a geração de renda e para a conservação do meio ambiente, sempre com a premissa de serem explorados de maneira sustentável. Para isso, são necessárias pesquisas acerca da biologia, da distribuição e da abundância reprodutiva do PFNM a ser explorado. O presente artigo tem, por objetivo, propiciar uma visão panorâmica dos métodos biométricos adequados para avaliar o estoque de recurso em pé, a dinâmica da produção, os componentes resultantes e as colheitas reais dos tipos principais de PFNM nos trópicos. Também são revistos estudos que buscam estimar o valor econômico dos produtos da floresta.

Palavras-chave: Produtos florestais não-madeiráveis; quantificação; valoração; manejo florestal.
\end{abstract}

\begin{abstract}
Quantification and valuation of non wood forest products. Now a day the reduction of biodiversity due to the increase of the deforestation has made people find ways to preserve forest remainders in Brazil and in the rest of the world. In this context, the non wood forest products (NWFP) became an alternative to assist the conservation of forests, once that, in the most of the cases, the extraction of NWFP doesn't involve degradation of the forest resources. Being thus, has grown the number of researches on the potential of the NWFP for incoming increase and preservation of the environment, always with the premise to be explored in a sustainable form. For this, its necessary studies concerning biology, distribution and reproductive abundance of the NWFP to be explored. The present article has the aim to provide a panoramic vision about the biometric methods used to evaluated the resource supply, the dynamic of production, the resultant components an the real harvest of the most important kinds of NWFP in the tropics. Also are reviewed studies that estimate the economical value of the forest products.

Keywords: Non wood forest products; quantification; valuation; forest management.
\end{abstract}

\section{INTRODUÇÃO}

$\mathrm{O}$ interesse sobre a perda da biodiversidade tropical em função do desflorestamento conduziu a um reconhecimento do valor dos produtos florestais não-madeireiros (PFNM) das florestas tropicais (MYERS, 1988; DE BEER; MCDERMOTT, 1989; PETERS et al., 1989).

Nesse contexto, Gentry; Blaney (1990), Prance (1987) e FAO (1995) comentam que esses produtos passaram a ser vistos como uma maneira viável para se explorar a riqueza biológica de florestas tropicais sem prejudicá-las e, ao mesmo tempo, estimular o desenvolvimento rural. 
Complementando, Nepstad; Schwartzman (1992) informam que, quando da extração de PFNM, a estrutura e a função da floresta não são alteradas e, na maioria dos casos, essa operação não envolve a degradação dos recursos naturais. Considerando tais fatos, o uso desses produtos foi introduzido como uma estratégia de conservação e de desenvolvimento florestal.

Cabe aqui mencionar que há uma proliferação dos estudos sobre o potencial dos PFNM para a geração de renda, como meio de envolver povos locais na gerência de florestas e para compartilhar dos benefícios que estas proporcionam, sempre considerando que os recursos, sejam eles animais ou vegetais, devam ser explorados em uma base sustentável. Entre essas pesquisas, podem-se citar as desenvolvidas por Menezes (2004) e Balzon (2006).

Um ponto que merece ser enfatizado é que, para serem sustentáveis, os níveis da colheita necessitam ser baseados em um bom conhecimento da biologia, da distribuição e da abundância reprodutiva das espécies da floresta (WICKENS, 1991). Tais informações podem ser obtidas a partir de fontes que incluam desde o conhecimento adquirido por povos indígenas até o inquérito científico formal.

De acordo com FAO (1995), pelo menos 150 PFNM têm significado no comércio internacional, representando um valor estimado de 11 bilhões de dólares em 1997. No Brasil, a renda gerada pelo extrativismo no período de 1995-1996 foi de aproximadamente 430 milhões de dólares, dos quais 322 milhões se referem aos produtos não-madeireiros (FAO, 1995).

De acordo com Soares et al. (2008), no Brasil e em todo o mundo, o potencial de mercado dos produtos florestais não-madeireiros cresce a cada dia, com o aumento da variedade de produtos tradicionais, tanto em função das florestas como do cultivo em sistemas agroflorestais. Contrapondo-se a esse potencial, percebe-se que, para alavancar a economia dos países em desenvolvimento, estudos sobre manejo de PFNM ainda são incipientes (SOARES et al., 2008).

Nesse sentido, o desafio é o desenvolvimento de estudos sobre o aprimoramento das formas de quantificação e projeção do valor dos recursos florestais não-madeireiros, de modo a integrar o capital natural na análise econômica, em que os métodos de valoração têm relevante papel. Pode-se, inclusive, ponderar que a necessidade de estimar valores para os ativos ambientais atenda às necessidades da adoção de medidas que visem à utilização sustentável do recurso (MARQUES; COMUNE, 1996).

O objetivo deste artigo de revisão é propiciar uma visão panorâmica dos métodos biométricos adequados para avaliação do estoque de recurso em pé, a dinâmica da produção, os componentes resultantes e as colheitas reais dos tipos principais de PFNM nos trópicos. Também são revistos estudos que buscam estimar o valor econômico dos produtos da floresta.

\section{METODOLOGIA DE QUANTIFICAÇÃO}

Considerando que PFNM podem propiciar tanto elementos provenientes da flora quanto da fauna, é necessário rever metodologias separadamente.

A revisão a respeito da avaliação quantitativa de PFNM revela uma gama de metodologias. Entretanto, em grande parte dos estudos realizados, percebe-se a utilização de técnicas sociais e de inventários.

As técnicas sociais envolvem entrevistas com os moradores e extratores da área estudada, para obtenção de informações sobre os costumes e conhecimentos locais, sendo de grande importância para o planejamento de atividades na região (GUNATILAKE, SENARATNE; ABEYGUNAWARDENA, 1993; POSEY, 1986; SANO; LIMA; CORREIA, 2008; RUIZ et al., 2002).

O inventário quantitativo, por sua vez, é definido como uma enumeração biométrica rigorosa da abundância e da distribuição de populações do recurso (BRAZ et al., 1995). O inventário é uma atividade importante que fornece os dados necessários sobre a qualidade e a disponibilidade dos recursos naturais, das atividades humanas e dos fenômenos naturais que afetam os recursos ambientais (KUNJEKU, 1997). Ainda de acordo com esse autor, o objetivo geral de um inventário é realizar o levantamento e a documentação dos componentes, da estrutura, da tendência espacial e do funcionamento básico dos elementos do ambiente, e de salientar que o ambiente, nesse contexto, não se restringe apenas aos fenômenos biofísicos, incluindo também os aspectos socioeconômicos e culturais.

Já Chenje (1997) considera um inventário como o processo de fazer observações singulares, não repetitivas. Quando as observações forem repetitivas, fala-se então de monitoria. Nesse último caso, o inventário forma a base para os programas futuros de monitoria e avaliação, devendo se proporem os indicadores mais apropriados para medir o grau de cumprimento de tais programas. 
Oltheten (1995) complementa argumentando que os termos inventário, monitoria e pesquisa tendem a subdividir artificialmente o processo geral de avaliação. Assim sendo, esse último termo é preferido neste artigo, não só porque engloba os primeiros, mas também porque tem mais sentido falar de avaliação participativa do que inventário participativo, por exemplo. Aliado a esse ponto, tem-se que um pressuposto importante é o de que a utilização da abordagem participativa cria um ambiente conducente à integração dos sistemas de conhecimento locais (local knowledge systems) no processo de planificação e implementação de projetos e programas de desenvolvimento. São quatro as condições que facilitam o planejamento e gestão do uso dos recursos florestais:

1) existência de uma organização local e um apoio comunitário forte;

2) sistemas apropriados de propriedade dos recursos (resource tenure systems);

3) repartição equitativa dos benefícios provenientes do patrimônio local;

4) sistemas de conhecimentos e de gestão locais.

O inventário florestal define-se como um sistema de coleta e análise de dados com o objetivo de avaliar a quantidade e a qualidade dos recursos florestais existentes numa área específica (CHIDUMAYO, 1997).

Sanquetta et al. (2006) conceituam inventário florestal como a atividade que visa avaliar, quantitativa e qualitativamente, a floresta alvo do manejo, sendo que este pode ser realizado mediante enumeração completa ou censo, isto é, com a medição de todos os indivíduos adultos ou mais convencionalmente com o emprego de técnicas de amostragem.

Chidumayo (1997), por sua vez, afirma que existem quatro objetivos ou justificativas gerais para se fazer inventários florestais:

1) planejamento do uso da terra;

2) planejamento do desenvolvimento florestal com ênfase num produto ou produtos florestais particulares;

3) estudos de viabilidade para uma indústria de produtos florestais;

4) planejamento florestal para determinar os volumes disponíveis, sua distribuição, utilização e regeneração dos produtos florestais, bem como a sua conservação.

Hansis (1998), em estudo realizado na região do noroeste dos Estados Unidos, na divisa com o Pacífico, utilizou-se de entrevistas informais tanto para avaliar as questões sociais das comunidades como para verificar questões geradas pelos exames de permissões para colher produtos da floresta. Esse autor também pesou os produtos, pois, segundo ele, a lembrança dos extrativistas nem sempre é confiável, porque eles dizem o que pensam que o pesquisador gostaria de ouvir. As entrevistas realizadas individualmente foram relacionadas às quantidades de produtos colhidos, à renda obtida por produto, à comercialização e à sustentabilidade da extração.

Em estudos desenvolvidos por Braz et al. (1995), utilizou-se o Inventário Florestal, considerando distribuição, abundância dos diferentes recursos e tipologia florestal. Nesse trabalho, foram também realizados levantamentos etnobotânicos, os quais caracterizaram as espécies segundo seu uso: alimentação, construção, medicinal e utensílios, entre outros.

Ruiz et al. (2002) também se utilizaram de entrevistas, usando a combinação de listagem, que consiste na elaboração de uma relação de espécies a partir de perguntas dirigidas a membros da comunidade sobre as plantas que utilizam e perguntas abertas sobre o uso para cada planta, ordenadas de acordo com o valor de importância das espécies quanto ao potencial de uso.

Em pesquisa feita na Amazônia, Reydon et al. (2002) utilizaram o método de Diagnóstico de Sistemas Agrários (DAS), que permitiu identificar os sistemas de produção existentes nas regiões. Para aplicação desse procedimento, foram realizados levantamento de campo, através da coleta de dados primários, além de se basear em outros estudos já desenvolvidos na região. Também com o objetivo de identificar os diversos aspectos da comercialização dos PFNM com potencial de mercado, foram entrevistados os produtores e empresas que atuam no local e que demandam PFNM.

\section{METODOLOGIA DE VALORAÇÃO}

Modelos econômicos, assim como métodos e princípios contábeis têm sido desenvolvidos para que se possam realizar análises econômicas do meio ambiente, porém são ainda pouco empregados, mesmo quando se tem urgência em utilizá-los e a consciência de que o desenvolvimento deve ser sustentável. A economia, a contabilidade nacional e a administração são campos de pesquisa que ainda caminham no sentido de incorporar a variável ambiental em suas análises. 
A necessidade de conceituar o valor econômico do meio ambiente e de desenvolver técnicas para estimar esse valor surgem, basicamente, do fato incontestável de que a maioria dos bens e serviços ambientais e das funções providas ao homem pelo ambiente não é transacionada pelo mercado. Pode-se, inclusive, ponderar que a necessidade de estimar valores para os ativos ambientais atende às necessidades da adoção de medidas que visem à utilização sustentável do recurso (MARQUES; COMUNE, 1996).

Cabe aqui mencionar que Merico (1996) afirma que os métodos de valoração ambiental procuram, de forma direta ou indireta, o valor dos bens e serviços ambientais, assim como de suas melhorias e degradações, possibilitando a incorporação dessas informações no processo de tomada de decisões da sociedade. A análise econômica torna-se mais completa quando incorpora aquilo que o mercado não quer ver, no caso os valores associados ao capital natural.

Assim, ressalta-se aqui a importância do desenvolvimento de estudos sobre o aprimoramento das formas de integrar o capital natural na análise econômica, no qual os métodos de valoração têm relevante papel.

Nesse contexto, Comune et al. (1995) salientam que o desenvolvimento sustentado seria a forma mais adequada para se tratar a problemática da degradação ambiental, pois incorpora, além do crescimento econômico, valores mais amplos de qualidade de vida de uma sociedade. Também de acordo com esse autor, nesse panorama, a economia ecológica contribui como o peso regulador da balança entre o meio ambiente e o desenvolvimento econômico, tentando resolver conflitos de interesse.

A economia ecológica é dinâmica, sistêmica e evolucionista. Seu foco principal é a relação do homem com a natureza e a compatibilidade entre crescimento demográfico e disponibilidade de recursos. Assim, ela é definida como um campo transdisciplinar que estabelece relações entre os ecossistemas e o sistema econômico e tem por objetivo agregar os estudos de ecologia e economia. Além disso, viabiliza extrapolar suas concepções convencionais, procura tratar a questão ambiental de forma sistêmica e harmoniosa e busca a formulação de novos paradigmas (CONSTANZA; DALY, 1991).

Serôa da Motta (1998) relata que a análise ambiental consiste em uma atividade interdisciplinar, que envolve aspectos econômicos e ecológicos para a obtenção do valor dos ecossistemas como um todo, exigindo cooperação e coordenação entre os grupos envolvidos. De acordo com Tognella (1995), devido ao possível esgotamento dos recursos naturais, surgiram vários estudos buscando o manejo e a conservação do meio ambiente, utilizando-se para isso, diversas metodologias que visam o valor intrínseco dos bens e serviços ambientais, expresso através dos processos de valoração econômica. Para Schweitzer (1990) ${ }^{1}$, citado por Vicent, Crawfor e Hoehn (1990), a valoração ambiental é essencial, pois se pretende que a degradação da grande maioria dos recursos naturais seja interrompida antes que ultrapasse o limite da irreversibilidade.

Pillet (1993) ressaltou que o valor econômico do ambiente passa pela revelação de preços não pagos pelo ambiente. Para esse autor, revelar preços não pagos significa que eles poderiam ou, até mesmo, deveriam ser pagos.

Conforme Finco (2002), a valoração econômica do meio ambiente surge quando da crescente preocupação mundial com a conservação dos recursos naturais. Essa preocupação deriva, sobretudo, do aumento da demanda pela qualidade dos bens e serviços gerados por esses recursos, ao mesmo tempo em que há uma enorme perda de bem-estar com a variação na quantidade e na qualidade desses serviços, por parte da geração presente, e pela preocupação com a geração futura. A valoração econômica aparece como uma ferramenta utilizada para atribuir valores aos bens e serviços providos pelo meio ambiente, como forma de captar os custos e benefícios oriundos das variações na quantidade e na qualidade desses bens e serviços.

Medeiros (2000) cita que os métodos de valoração buscam suprir a inexistência de mercados e preços para as externalidades derivadas de bens públicos constituídos por recursos naturais. Já as técnicas de valoração são classificadas, segundo a Economia do Meio Ambiente e dos Recursos Naturais, em métodos da função de produção - método da produtividade marginal e de mercados de bens substitutos e métodos da função de demanda - métodos que utilizam mercados de bens complementares, como métodos dos preços hedônicos e custo de viagem, e valoração contingente.

Ainda pelo relato de Serôa da Motta (1998), os métodos de função de produção analisam casos em que o recurso ambiental está associado à produção de um recurso privado e geralmente assumem que as variações na oferta do recurso ambiental não alteram os preços de mercado. Por outro lado, os métodos

\footnotetext{
${ }^{1}$ SCHWEITZER, J. Economics, conservation and development: a perspective from USAID. In: VICENT, J. R.; CRAWFOR, E. W.; HOEHN, J. P. Valuing environmental benefits in developing countries: proceedings. East Lansing: Michigan State Univ., 1990.
} 
de função de demanda admitem que a variação da disponibilidade do recurso altere o bem-estar das pessoas e, portanto, é possível identificar as medidas de disposição a pagar ou a receber das pessoas em relação a essas variações.

Para Silveira $(2002)^{2}$, citado por Braga; Oliveira; Abdallah (2008), uma grande parcela dos economistas considera que a inserção da problemática ambiental na economia ocorre a partir de alguns fundamentos, cujos princípios podem ser resumidos na concepção de mercado, na medida em que os agentes econômicos procuram maximizar suas utilidades, tendo o preço como indicador do grau de escassez das amenidades ambientais. Dessa forma, os problemas relativos ao meio ambiente são considerados falhas de mercado, externalidades negativas, o que requer a correção através do ato de valorar os danos ambientais.

De acordo com Mattos; Filho; Mattos (2000), a proposta de avaliação monetária dos recursos naturais surge com objetivo de mostrar o valor econômico de certo tipo de ambiente e o prejuízo irrecuperável que pode ocorrer caso este seja destruído.

Segundo Marques; Comune (1996) e Mattos; Filho; Mattos (2000), os métodos de valoração ambiental podem ser classificados em diretos e indiretos, sendo os métodos diretos baseados em informações de mercados já existentes e os indiretos em mercados hipoteticamente criados, destacando-se os métodos da valoração contingente, custo de viagem e mercado substituto ou preço hedônico. Os métodos indiretos são assim chamados porque seus procedimentos diferem no sentido de não procurarem medir o estado das preferências diretamente.

Conforme Godoy; Bawa (1993), o estudo da avaliação econômica dos PFNM deve ser direcionado para o ponto da floresta onde as populações locais negociam ou consomem os bens, em detrimento da avaliação do valor dos bens da floresta para a economia regional, nacional ou mundial. Devem-se omitir estudos que apresentem pouca informação para permitir o cálculo do lucro anual por hectare.

Vários estudos mostram que o valor do lucro líquido varia amplamente, oscilando entre cerca de US\$1 até cerca de US\$ 420/ha/ano. A variação pode ser explicada pela diversidade ecológica e econômica entre os locais amostrados dos diferentes estudos, pela diferença dos métodos e pressupostos utilizados e pelos diferentes produtos estudados. Apesar de se estudarem os mesmos bens, avaliações independentes conduzidas por períodos de tempo semelhantes têm produzido diferentes resultados.

Godoy; Bawa (1993) afirmam que, para estimar o valor econômico dos produtos florestais nãomadeireiros da floresta tropical, tomaram como base, além de suas experiências, a literatura antropológica sobre caçadores e extrativistas, exames de métodos em antropologia social e conferências com alguns dos principais pesquisadores do uso das plantas e animais da floresta tropical.

Alguns especialistas, entre eles Godoy; Lubowski; Markandya (1993), usam despesas de trabalho ou energia como uma forma de medir o valor em sociedades sem mercado. Todavia, essas duas medidas podem gerar diferentes resultados, além de que nenhuma delas é consistente com a teoria moderna de valoração aplicada a essa área. Diante dessa realidade, segundo esses autores, para se adquirir uma medida precisa dos custos marginais de extração e processos de PFNM, deve-se calcular o custo dos materiais, usando o tempo levado para achar, extrair, processar e transportar os bens.

Esse método requer entrevistas com os coletores sobre a proporção do tempo dedicada às diferentes atividades durante o dia, a dedicação e o acompanhamento direto do pesquisador para que se obtenham as estimativas mais confiáveis do custo total do trabalho. Entretanto, é bastante dificultado pelas medições inadequadas das quantidades extraídas, custos e tempo (GODOY; LUBOWSKI; MARKANDYA, 1993).

Os métodos de valoração mais simples de aplicar são aqueles que se baseiam em preços de mercado e quantidade e qualidade de informação relacionada, para derivar valores totais, sendo direcionados, em sua maioria, a estudos de custo-benefício (IZCO; BURNEO, 2003). Ainda de acordo com esses autores, quando são utilizados preços de mercado com o propósito de realizar valorações financeiras, é importante determinar os preços corretos para os bens e serviços de cada alternativa de uso do solo. Em alguns casos, é necessário ajustar os preços (preços-sombra), devido à existência de imperfeições de mercado.

Para Godoy; Bawa (1993), o melhor método de valoração é fazer uso do preço já existente para o produto, ou do que prevaleça em mercados relacionados. Outro método é substituir o valor por um

\footnotetext{
${ }^{2}$ SILVEIRA, F. C. Jornal A Gazeta. Set. 2002.
} 
produto compatível, ou seja, o método comparativo de dados de mercado é aquele que define o valor através da comparação com dados de mercado semelhantes quanto às características intrínsecas e extrínsecas dos bens ou serviços. As características e os atributos dos dados pesquisados que exercem influência na formação dos preços e, consequentemente, no valor, devem ser ponderados por homogeneização ou por inferência estatística, respeitando os níveis de rigor definidos pela norma. Uma condição fundamental para a aplicação deste método é a existência de um conjunto de dados que possa ser tomado, estatisticamente, como amostra.

Moreira Filho et al. (1993) comentam que a pesquisa de mercado é a tarefa fundamental do método comparativo, pois é através dela que será formada a amostra e também serão coletadas as informações que permitirão a identificação e seleção das variáveis a serem consideradas na avaliação.

No trabalho de Braz et al. (1995), realizado na Floresta Estadual do Antimary, desenvolveram-se estudos de botânica econômica, buscando-se informações relacionadas a produtos como óleos, sabões, xampus e cremes possíveis de serem elaborados com espécies nativas. O principal objetivo desse estudo foi avaliar a viabilidade da comercialização de tais produtos.

Outro método citado por inúmeros estudiosos do assunto, em que se destacam Serôa da Motta (1998) e Benakouche; Cruz (1994) $)^{3}$, citados por Hildebrandt (2001), é o de valoração contingente (Contingent Valuation Method), no qual os usuários dos produtos são questionados sobre a sua disposição a pagar pelo bem. Esse método consiste na aplicação de questionários que permitam revelar as preferências dos consumidores e então captar as disposições a pagar (DAP) dos indivíduos pelo uso ou conservação de um bem ou ambiente. O procedimento metodológico é a coleta, elaboração e análise dos dados. Os questionários são empregados para se obter a máxima DAP para ter esse benefício, a mínima compensação de ficar sem ele ou, ainda, a DAR (Disposição a Receber) por algum malefício. Segundo Borger (2001), para que o questionário a ser aplicado no processo de entrevistas tenha sucesso, devem existir algumas condições, tais como a familiaridade do entrevistado com os benefícios que geram o projeto, a explicitação de um instrumento de pagamento conhecido ou aceitável pelo entrevistado e a fixação do montante a pagar.

Os indivíduos podem indicar suas escolhas ou preferências de diversas formas. Uma delas é responder ao questionário relatando se aceita ou não a valoração de um serviço, se este for a custo preestabelecido. Outra alternativa é perguntar às pessoas se estão dispostas a pagar por determinado bem ou serviço. Esses dois tipos de questões podem ser combinados em um questionário, com o objetivo de captar a informação sobre a valoração ambiental (BRAGA; OLIVEIRA; ABDALLAH, 2008).

Comune et al. (1995) salientaram que uma das vantagens desse tipo de metodologia consiste justamente em produzir estimativas de valores que não poderiam ser obtidos por outros meios. O objetivo da valoração contingente é de deduzir avaliações ou ofertas bastante semelhantes àquelas que seriam reveladas se o mercado existisse. Os questionários sobre o bem ou recurso a ser valorado deve descrevêlo claramente para que os entrevistados tenham conhecimento do que está sendo valorado.

\section{CONCLUSÕES}

Os métodos de quantificação apresentam-se bastante objetivos quanto ao processo e técnicas de aplicação, diferentemente da valoração econômica dos produtos florestais não-madeireiros, a qual não possui uma clasificação rígida, podendo se utilizar de diversos enfoques, dependendo do propósito.

Sendo assim, além da dificuldade na mensuração de custos, quantidades extraídas e preços, os estudos até então realizados não podem ser diretamente comparados, uma vez que se utilizam de métodos diferentes na sua concepção e o valor econômico está diretamente relacionado à biodiversidade de cada local.

Constata-se que as demandas por pesquisas que esclareceram a maneira de se contabilizar os recursos naturais são grandes, e que somente pesquisas não bastam, pois se estas não forem aplicáveis não terão sentido. Assumindo-se a postura de um crescimento sustentável, a aplicação de contabilização e métodos de avaliação econômica dos recursos naturais torna-se demanda imprescindível, que aos poucos pode ultrapassar os meios acadêmicos e tomar lugar em mecanismos de organizações públicas e privadas.

${ }^{3}$ BENAKOUCHE, R.; CRUZ, R. S. Avaliação monetária do meio ambiente. São Paulo: Makron Books, 1994. 198 p. 


\section{REFERÊNCIAS}

BALZON, D. R. Avaliação econômica dos produtos florestais não madeiráveis na área de proteção ambiental - APA de Guaratuba - Paraná. Curitiba. Tese (Doutorado em Ciências Florestais) - Setor de Ciências Agrárias, Universidade Federal do Paraná, Curitiba, 2006.

BORGER, F. G. Responsabilidade social: efeitos da atuação social na dinâmica empresarial. Tese (Doutorado em Administração) - Faculdade de Economia, Administração e Contabilidade, Universidade de São Paulo, São Paulo, 2001.

BRAGA, P. L. S.; OLIVEIRA, C. R.; ABDAllAH, P. R. Aplicação do Método de Valoração Contingente no Parque Nacional da Lagoa do Peixe - RS. Disponível em: <http://www.ceema.furg.br/paolaartuni camp.pdf>. Acesso em: 20/02/2008.

BRAZ, E. M.; FIGUEIREDO, E. O.; OLIVEIRA, L. C.; FERREIRA, L. A.; SILVA, Z. A. G. P. G. Manejo dos Produtos Florestais Não Madeireiros da Floresta Estadual do Antimary: a busca de um modelo. Rio Branco: FUNTAC, 1995.

CHENJE, M. (Ed). Working glossary for the environment report. State of the Environment Report (SOER). [S.1.]: SADC-IMERCSA, 1997. (Occasional Paper, n. 3).

CHIDUMAYO, E. N. Miombo ecology and management: an introduction. Stockholm: IT Publications and Stockholm Environment Institute, 1997.

COMUNE, A. E.; GRASSO. M.; TOGNELLA, M. M. P.; SCHAEFFER, Y. N. Aplicação de Técnicas de Avaliação Econômica ao Ecossistema Manguezal. In: Valorando a natureza: análise econômica para o desenvolvimento sustentável. Rio de Janeiro: Campus, 1994. cap. 7.

CONSTANZA, R.; DALY, H. Toward ecological economics: modeling ecological. New York: Elsevier, 1991.

DE BEER, J. H.; MCDERMOTT, M. J. Economic value of non-timber forest products in south-east Asia. Amsterdam: The Netherlands Committee for IUCN, 1989.

FAO. Non-wood forest products for rural income and sustainable forestry. Rome. 1995. (Non-wood Forest Products, n. 7).

FINCO, M. V. A. Instrumentos econômicos como ferramenta de valoração ambiental: caso de estudo: Praia do Cassino, Rio Grande / RS, Brasil. 94 p. Monografia (Ggraduação em Ciências Econômicas) - Fundação Universidade Federal do Rio Grande, Rio Grande, 2001.

GENTRY, A. H.; BLANEY, C. L. Tropical forestry. Western-Wildlands, Missoula, v. 16, n. 1, p. 2-24, 1990.

GODOY, R. A.; BAWA, K. S. The economic value and sustainable harvest of plants and animals from the tropical forest: assumptions, hypotheses, and methods. Economic Botany, New York, v. 47, n. 3, p. 215- 219, 1993.

GODOY, R.; LUBOWSKI, R.; MARKANDYA, A. Um método para a avaliação econômica de Produtos Florestais Não Madeireiros. Economic Botany, New York, v. 47, n. 3, p. 220-233, 1993.

GUNATILAKE, H. M.; SENARATNE, D. M. A. H.; ABEYGUNAWARDENA, P. Role of nom-timber products in the economy of peripheral communities of Knuckles National Wilderness Area of Sri Lanka: a Farming Systems Approach. Economic Botany, New York, v. 47, n. 3, p. 275-281, 1993.

HANSIS, R. A political ecology of picking: non-timber forest products in the Pacific Northwest. Human Ecology, London, v. 26, n. 1, p: 49-68, 1998.

HILDEBRANDT, E. Avaliação econômica dos benefícios gerados pelos parques urbanos: estudo de caso em Curitiba - PR.. Tese (Doutorado em Ciências Florestais) - Setor de Ciências Agrárias, Universidade Federal do Paraná, Curitiba, 2001. 
ICZO, X.; BURNEO, D. Ferramentas para a Valoração e Manejo Florestal Sustentável dos Bosques Sul-Americanos. Resumo Executivo UICN, 2003. Disponível em: <www.sur.iucn.org/publicaciones/documentos/publicaciones/348.pdf>. Acesso em: 20/02/2008.

JOHNS, A. D. Effects of 'selective' timber extraction on rain forest structure and composition and some consequences for frugivores and folivores. Biotropica, Washington, DC, v. 20, n. 1, p. 31-37, 1988.

KUNJEKU, E. et al. Research, inventory and monitoring. In: CHENJE, M.; SOLA, L. ; PALECZNY, D. The state of Zimbabwe's environment. Zimbabwe: Government of the Republic of Zimbabwe, Ministry of Mines, Environment andTourism, Harare, 1998.

MAY, P. H.; MOTTA, R. S. Valorando a natureza: análise econômica para o desenvolvimento sustentável. Rio de Janeiro: Campus, 1994.

MARQUES, J. F.; COMUNE, A. E. A teoria neoclássica e a valoração ambiental. In: ROMEIRO, A. R.; REYDON, B. P.; LEORNARDI, M. L. A. Economia do meio ambiente. Campinas: Unicamp, 1997. p. $21-42$.

MATTOS, K. M. C.; FILHO, N. J. F.; MATTOS, A. Uma abordagem conceitual sobre a valoração econômica. Disponível em: 〈http://www.cpap.embrapa.br/agencia/congresso/Socio/mattos-043.pdf> Acesso em: 16/01/2002.

MEDEIROS, C. P. S. Importância econômica da biodiversidade: uma análise social de custo-benefício para o Parque Nacional do Superagüi no Litoral Norte do Estado do Paraná, Brasil. Trabalho apresentado no V. Encontro Brasileiro de Ecossistemas, 2000.

MENEZES, R. S. de. A importância da reserva legal na geraçăo de renda de pequenos produtores rurais : estudo de caso no Estado do Acre, Amazônia. 104 f. Dissertação (Mestrado em Engenharia Florestal) - Setor de Ciências Agrárias, Universidade Federal do Paraná, Curitiba, 2004.

MOREIRA FILHO, I. I.; FRAINER, J. I.; MOREIRA, R. M. I. Avaliação de bens por estatística inferencial e regressões múltiplas, teoria e aplicações. 2. ed. [S.l.: s.n.], 1993. v.1.

MOTTA, R. S. Contabilidade ambiental, teoria, metodologia e estudos de caso no Brasil. Rio de Janeiro: IPEA, 1995.

MOTTA, R. S. Manual para valoração econômica de recursos ambientais. Brasília, DF: Ministério do Meio Ambiente, dos Recursos Hídricos e da Amazônia Legal, 1998.

MYERS, N. Tropical forests: much more than stocks of wood. Journal of Tropical Ecology, Cambridge, v. 4, p. 209-221, 1988.

NEPSTAD, D. C.; SCHWARZMA, S. Non-timber products from tropical forests: evaluation of a conservation and development strategy. New York: New York Botanical Garden, 1992.

OLTHETEN, T. M. P. Participatory approaches to planning for community forestry. Rome: FAO, FTPP, 1995.

PETERS, C. M.; GENTRY, A. H.; MENDELSOHN, R. O. Valuation of an Amazonian Rainforest. Nature, London, n. 339, p. 655-656, 1989.

PILLET, G. Economia ecológica: introdução à economia do ambiente e recursos naturais. São Paulo: Instituto Piaget, 1993.

PRANCE, G. T.; BALEE, W.; BOOM, B. M.; CARNEIRO, R. L. Quantitative ethno botany and the case of conservation in Amazonia. Conservation Biology, Boston, v. 1, n. 4, p. 296-310, 1987.

POSEY, D. A. Manejo da floresta secundária, capoeiras, campos e cerrados (Kayapó). In: RIBEIRO, D. (Ed.). Suma etnológica brasileira. Petrópolis: Vozes/FINEP, 1986. v, 1, p. 173-188. 
REYDON, B. P.; MACIEL, R. C. G. Valoração econômico-ambiental de uma alternativa produtiva na Reserva "Chico Mendes". Instituto de Economia, Unicamp, Campinas. Disponível em: <http://www.eco.unicamp.br/projetos/gestao_ambiental/gestaoambiental.html >. Acesso em: 18/02/2008.

REYDON, B. P.; SCHLOGL, A. K. S. B.; HENRY, G. Produtos florestais não madeireiros da Amazônia: limites e perspectivas enquanto alternativa para o desenvolvimento sustentável da região. Floresta, Curitiba, n.esp., p. 127-133, set. 2002.

ROMEIRO, A. R. et al. Economia do meio ambiente: teoria, políticas e a gestão de espaços regionais. Campinas: UNICAMP, 1996.

RUIZ, R. C.; COSTA, L. S.; SILVEIRA, M.; BROWN, I. F. Seleção de espécies vegetais com potencial de uso, para estudos ecológicos e manejo, em florestas no oeste da Amazônia. New York: The New York Botanical Garden, 2002. 13 p.

SANO, S. M.; LIMA, I. L. P.; CORREIA, J. R. Avaliação Participativa do Extrativismo de Mangaba (Hancornia speciosa GOMES) em Rio Pardo de Minas, MG. Disponível em: <http://www.cnpat.embrapa.br/sbsp/anais/trab_format_pdf/89.pdf >. Acesso em: 20/03/2008.

SANQUETTA, C. R.; MATTEI, E.; CORTE, A. P. D.; SANTOS, A. J.; MULlER, A. C.; STOLLE, L.; PIRES, P. T. L.; BALBINOT, R. Perspectivas de recuperação e manejo sustentável das florestas de Araucária. Curitiba: Multigraphic. 2006, 264 p.

SILVA, J. A. Análise quali-quantitativa da extração e do manejo dos recursos florestais da Amazonia brasileira: uma abordagem geral e localizada (Floresta Estadual do Antimari - AC). 153 f. (Tese de Doutorado) - Setor de Ciências Agrárias, Universidade Federal do Paraná, Curitiba, 1996.

SOARES, T. S.; FIEDLER, N. C.; SILVA, J. A.; GASPARINI JÚNIOR, A. J. Produtos Florestais Não Madeireiros. Revista Científica Eletrônica de Engenharia Florestal. Ed. 11. Fev. 2008. 7 p. Disponível em: 〈http://www.revista.inf.br/florestal/pages/artigos/ARTIGO06.pdf>. Acesso em: 28/03/2008.

VICENT, J. R.; CRAWFOR, E. W.; HOEHN, J. P. ed. Valuing environmental benefits in developing countries: proceedings. East Lansing: Michigan State University, 1990.

TOGNELLA, M. M. P. Valoração econômica: estudo de caso para o ecossistema manguezal Bertioga e Cananéia. 161 p. Dissertação (Mestrado) - Instituto Oceanográfico, Universidade de São Paulo, São Paulo, 1995.

WICKENS, G. E. El desarollo de los productos forestales no madereros: principios de ordenacion. Unasylva, Roma, n. 165, v. 42, p. 4-8, 1991. 